\title{
Adventitial lymphatics of internal carotid artery in healthy and atherosclerotic vessels
}

\author{
Katarzyna Drożdż ${ }^{1}$, Dariusz Janczak², Piotr Dzięgiel ${ }^{3}$, Marzena Podhorska ${ }^{3}$, \\ Dariusz Patrzałek $^{2}$, Piotr Ziółkowski ${ }^{4}$, Ryszard Andrzejak ${ }^{1}$, Andrzej Szuba ${ }^{1}$
}

\author{
${ }^{1}$ Department of Internal Medicine; ${ }^{2}$ Department of Vascular, General and Transplantation Surgery; \\ ${ }^{3}$ Department of Histology; ${ }^{4}$ Department of Pathology, \\ Wrocław University of Medicine, Pasteura 1, 50-367 Wrocław, Poland
}

\begin{abstract}
Objectives. Presence of lymphatics in adventitia of major arteries remains controversial. Presence of lymphatics in adventitia of internal carotid artery was not documented and its relation to atherosclerosis was not studied. The aim of our study was to evaluate presence of lymphatic vessels in adventitia of internal carotid artery in healthy and atherosclerotic arteries. Methods. Fragments of arterial wall of internal carotid artery were obtained during the surgical eversion endarterectomy in 15 patients with internal carotid artery stenosis and 2 healthy organ donors. 21 arteries were studied. Patients age ranged from 56 to 77 years. Fragments of arterial wall were embeded in paraffin. Lymphatics of arterial adventitia were visualized with immunohistochemistry using LYVE-1 and anty-podoplanin antibodies. Results. The lymphatic vessels were visualized in adventitia of 20 carotid arteries. The serial sections have revealed that both LYVE-1 and podoplanin have identical specificity for lymphatic endothelium Number of lymphatics in adventitia significantly correlated with thickness of intima $(\mathrm{p}<0.046)$. Conclusions. Lymphatics are present in adventitia of internal carotid artery. Number of adventitial lymphatics increases with severity of atherosclerosis measured as intimal thickness.
\end{abstract}

Key words: vasa vasorum, lymphagiogenesis, lymphatic system

\section{Introduction}

The atherosclerosis is currently seen as a chronic inflammatory process with infiltration of mononuclear lymphocytes into the intima, local expansion of vascular smooth muscle cells, and accumulation of extracellular matrix [1]. Vascular adventitia for a long time was considered mainly a supportive tissue providing nutrients for the vascular wall, however its function in arterial pathology was suspected $[2,3]$. Recently many studies point at the role of the adventitia in initiation and development of atherosclerosis [4,5]. Adventitial vasa vasorum (VV) were found to proliferate in response to arterial injury and progress of atherosclerosis [6,7]. Lymphatics vessels are present in arterial adventitia, but their exact function remains unclear. Recently $\mathrm{Xu}$ and coworkers suggested that adventitial lymphatics play an important role in development of

Correspondence: A. Szuba, Dept. of Internal Medicine,

Wrocław University of Medicine, Pasteura 1 Str., 50-367

Wrocław, Poland; tel.: (+4871) 7842528, fax.: (+4871) 7840954,

e-mail:szubaa@yahoo.com, anszuba@gmail.com atherosclerosis enhancing activation and accumulation of inflammatory cells[8].

The lymphatic system regulates interstitial fluid balance, takes part in lipid absorption and lipid metabolism, and is crucial for development immune response. Adventitial lymphatics might be involved in reverse cholesterol and lipid transport from the arterial wall $[9,10]$ and in transport immune cells to the regional lymphnodes. In response to inflammation lymphatic vessels can proliferate. Lymphangiogenesis was detected in corneal inflammation [11], inflammatory neoplasms [12] and inflammatory bowel diseases [13]. Lymphangiogenesis in adventitia may accompany inflammation in atherosclerotic arteries.

The aim of our study was to detect lymphatic vessels in adventitia of internal carotid arteries and to analyse possible relationship with severity of atherosclerosis.

\section{Materials and Methods}

Patients. The fragments of internal carotid artery were collected during internal carotid surgery (eversion endarterectomy) in 15 patients ( 3 women and 12 men, 4 patients had bilateral endarterec- 

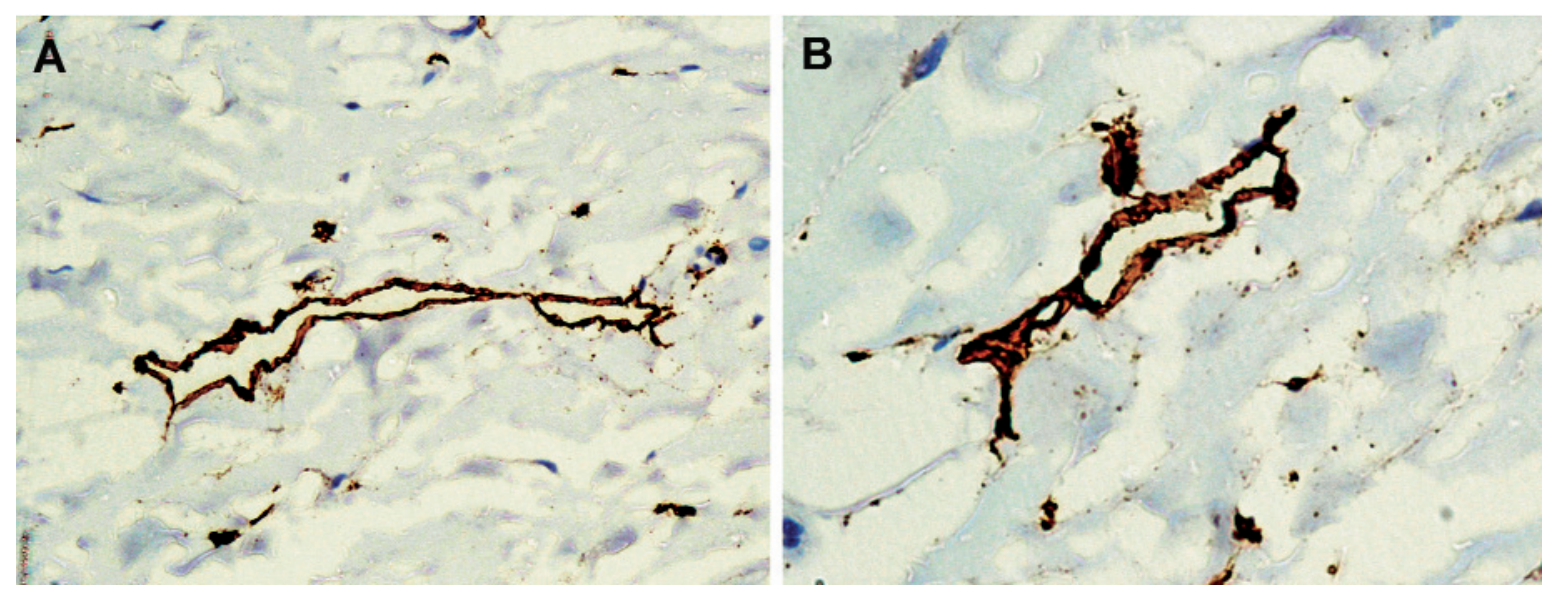

Fig. 1. Adventitial lymphatic vessels of internal carotid artery (anti-LYVE-1 Ab). Magnification $\times 200($ A) and $\times 400($ B).

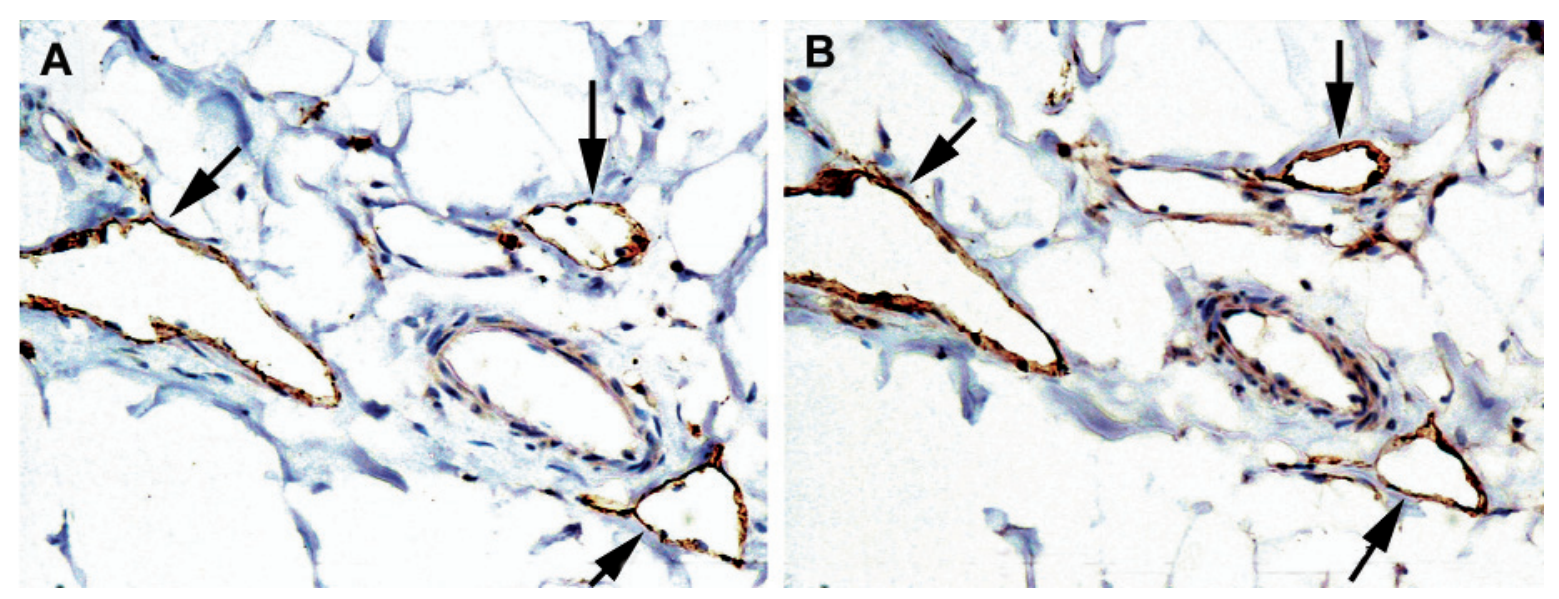

Fig. 2. Serial sections of internal carotid artery adventitia with lymphatic vessels. A. anti-LYVE-1 staining; B. anti-podoplanin staining. Black arrows point at lymphatics (magnification $\times 200$ ).

tomy) with symptomatic carotid artery stenosis over $70 \%$. Patients age ranged from 56 to 77 years. Additionally fragments of healthy internal carotid artery were harvested from 2 young (24 and 27 years old) organ donors. Altogether, samples of 21 carotid arteries were obtained for further studies. The study was approved by the Bioethical Committee of the Wrocław Medical University. The surgeries were performed at the Department of Vascular, General and Transplantation Surgery, Wrocław Medical University.

Tissue preparation. All fragments of arterial wall were initially fixed in $10 \%$ buffered formaldehyde solution, then embedded in paraffin and cut into $5 \mu \mathrm{m}$ sections. Paraffin sections from all 21 arteries were stained with hematoxylin and eosin for histological evaluation.

Immunohistochemistry. Immunohistochemical staining of lymphatic vessels was performed using specific antibodies for lymphatic endothelium. Paraffin sections were deparaffinized, dehydrated, and pre-treated with Target Retrieval Solution (DakoCytomation) at $95^{\circ} \mathrm{C}$ for $20 \mathrm{~min}$. After wash in Tris-buffered saline (TBS), the sections were treated with $3 \% \mathrm{H}_{2} \mathrm{O}_{2}$ for $10 \mathrm{~min}$. Subsequently after wash in distilled $\mathrm{H}_{2} \mathrm{O}(10 \mathrm{~min})$ and PBS $(5 \mathrm{~min})$ the sections were incubated with mouse monoclonal antibodies against LYVE-1 and podoplanin (RELIATech GmbH, Germany), diluted 1:200, for $60 \mathrm{~min}$ at room temperature. The serial sections were made for both podoplanin and the LYVE-1 antibodies. For all slides, a wash in TBS was followed by treatment with peroxidase-labeled polymer conjugated to goat anti-rabbit or anti-mouse immunoglobulins (Envision+kit; DakoCytomation, Denmark) for $30 \mathrm{~min}$ at room temperature. The immunostaining was visualized with diaminobenzidine tetrahydrochloride (DAB) and then counterstained with hematoxylin. In each case the negative control was included with Primary Negative Control (DakoCytomation, Denmark).

Light microscopy. The thickness of intima was measured under magnification $\times 100$, with Olympus BX 41 light microscope using the visual mode analySIS 3.2 software for computer. Intimal thickness served as a marker severity of atherosclerosis. For evaluation of lymphatic vessel number, slides were scanned with the Olympus BX 41 light microscope at $\times 200$ and then at $\times 400$ magnification by two different investigators. The morphological hallmarks for lymphatic vessel were: positive reaction with LYVE-1 and podoplanin antibodies, a thin vessel wall with irregular or collapsed lumen and no red blood cells and inward handed nucleus. We have examined the cross section of internal carotid artery and we have counted all lymphatic vessels in each cross section. The pictures were taken of each found lymphatic vessel.

Statistical analysis. Statistical analysis was performed with Statistica 5.1 PL software (StatSoft, Cracow, Poland). Mann-Whitney, 


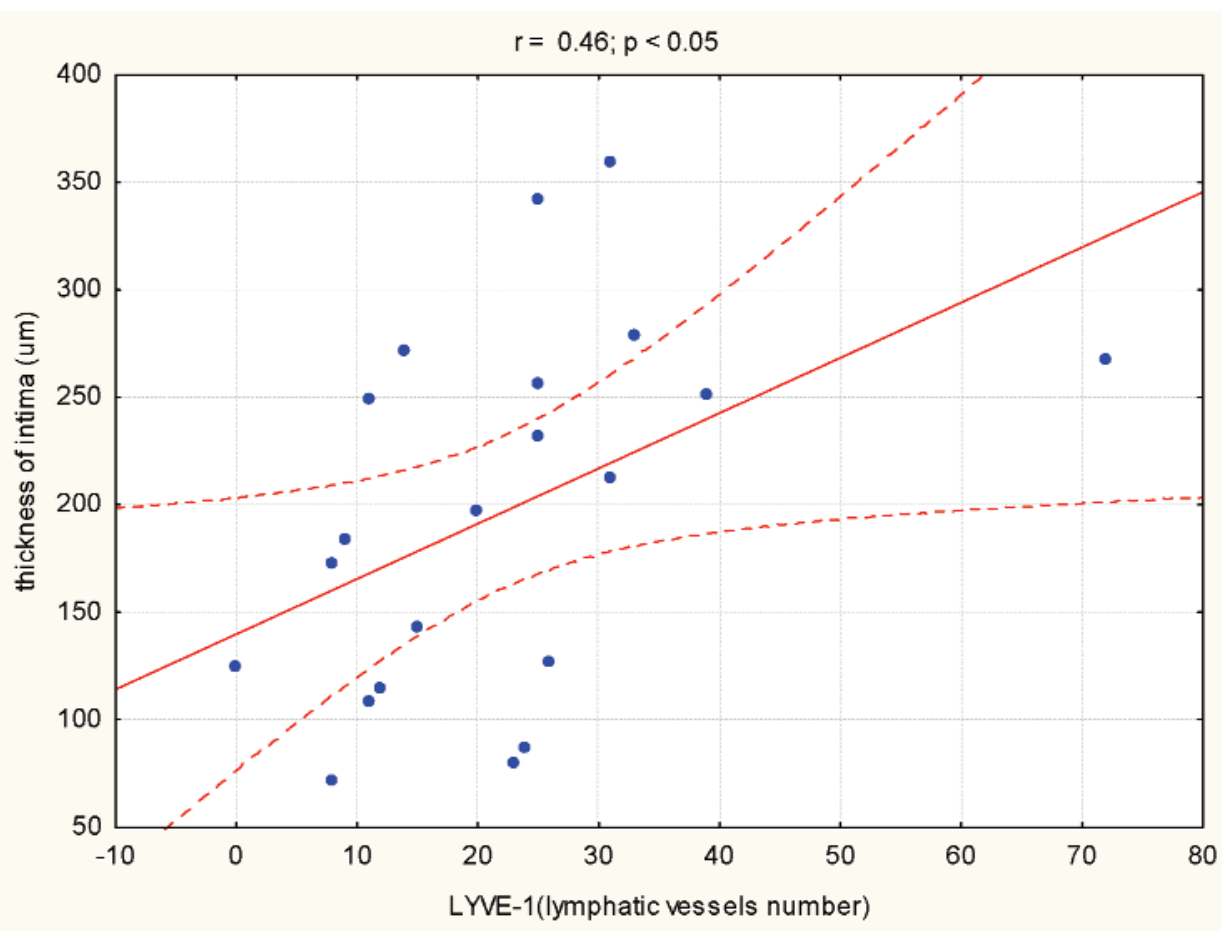

Fig. 3. Correlation between thickness of intima and number of lymphatic vessels in adventitia of internal carotid artery; $\mathrm{n}=21, \mathrm{r}=0.46$, $\mathrm{p}<0.05$.
F-Cox, Chi-square, and Spearman's correlation tests were performed. The differences were considered significant at $\mathrm{p}<0.05$.

\section{Results}

The lymphatic vessels were visualized by immunohistochemical reactions in 20 samples. There was one sample without visualized lymphatics. The lymphatics were found mainly in adventitia but also in periadventitia (Fig. 1). The serial sections have revealed that both LYVE-1 and podoplanin have identical specificity for lymphatic endothelium (Fig. 2). Degree of atherosclerosis severity was reflected by increased number of lymphatics in arterial adventitia (Fig. 3).

\section{Discussion}

The salient findings of our study are: confirmation of lymphatic vessels presence in adventitia of internal carotid arteries in humans and significant increase in adventitial lymphatic vessels number with progression of atherosclerosis.

Lymphatic vessels were detected in arterial adventitia of animal and human arteries by several authors [10,14-17]. However, there are reported differences between human an animal arteries. Adventitial lymphatics were found in coronary arteries in dogs [18] but not in humans [19]. Also different arteries may have different characteristics of adventitial vasa vasorum [20]. Therefore our finding of lymphatic vasa vasorum in human internal carotid adventitia is new and important. We have not detected adventitial lymphatics in one studied sample, where the specimen was almost completely lacking adventitia.

We have also found that increased thickness of arterial intima - a marker of atherosclerosis severity - is accompanied by increased number of lymphatics in adventitia. We believe that it might reflect inflammatory lymphangiogenesis in atherosclerotic arteries. Inflammation is a potent stimulus of lymphangiogenesis $[21,22]$ and inflammatory reaction described in adventitia of atherosclerotic arteries [5,23].

Our findings indicate that adventitial lymphatics play an important role in arterial wall metabolism and pathogenesis of atherosclerosis.

Further studies are necessary to elucidate function of adventitial lymphatics in atherosclerotic process.

Acknowledgements: The study was supported by the Wroclaw Medical University grant number 1047

\section{References}

[1] Fan J, Watanabe T. Inflammatory reactions in the pathogenesis of atherosclerosis. J Atheroscler Thromb. 2003;10:63-71.

[2] Heistad DD, Marcus ML. Role of vasa vasorum in nourishment of the aorta. Blood Vessels. 1979;16:225-238.

[3] Crotty TP. The role of vasa vasorum in atherosclerosis. Med Hypotheses 1989; 28: 233-243.

[4] Maiellaro K, Taylor WR: The role of the adventitia in vascular inflammation. Cardiovasc Res. 2007;75:640-648.

[5] Xu F, Ji J, Li L, Chen R and Hu WC. Adventitial fibroblasts are activated in the early stages of atherosclerosis in the apolipoprotein E knockout mouse. Biochem Biophys Res Commun. 2007;352:681-688. 
[6] Yamashita A, Shoji K, Tsuruda T, Furukoji E, Takahashi M, Nishihira K, Tamura S and Asada Y. Medial and adventitial macrophages are associated with expansive atherosclerotic remodeling in rabbit femoral artery. Histol Histopathol. 2008; 23:127-136.

[7] Langheinrich AC, Michniewicz A, Sedding DG, Walker G, Beighley PE, Rau WS, Bohle RM and Ritman EL. Correlation of vasa vasorum neovascularization and plaque progression in aortas of apolipoprotein E(-/-)/low-density lipoprotein(-/-) double knockout mice. Arterioscler Thromb Vasc Biol. 2006;26:347-352.

[ 8] Xu X, Lin H, Lv H, Zhang M and Zhang Y. Adventitial lymphatic vessels - an important role in atherosclerosis. Med Hypotheses. 2007;69:1238-1241.

[9] Nanjee MN, Cooke CJ, Wong JS, Hamilton RL, Olszewski WL and Miller NE. Composition and ultrastructure of size subclasses of normal human peripheral lymph lipoproteins: quantification of cholesterol uptake by HDL in tissue fluids. J Lipid Res. 2001;42:639-648.

[10] Jellinek H. Transport in the vessel wall in early changes of arteriosclerosis. Z Gesamte Inn Med. 1978;33:599-601.

[11] Cursiefen C, Maruyama K, Jackson DG, Streilein JW and Kruse FE. Time course of angiogenesis and lymphangiogenesis after brief corneal inflammation. Cornea. 2006;25:443447.

[12] Van der Auwera I, Van den Eynden GG, Colpaert CG, Van Laere SJ, van Dam P, Van Marck EA, Dirix LY and Vermeulen PB. Tumor lymphangiogenesis in inflammatory breast carcinoma: a histomorphometric study. Clin Cancer Res. 2005;11:7637-7642.

[13] Kaiserling E, Krober S and Geleff S. Lymphatic vessels in the colonic mucosa in ulcerative colitis. Lymphology. 2003; 36: 52-61.

[14] Sacchi G, Weber E and Comparini L. Histological framework of lymphatic vasa vasorum of major arteries: an experimental study. Lymphology. 1990;23:135-139.
[15] Nakano T, Nakashima Y, Yonemitsu Y, Sumiyoshi S, Chen YX, Akishima Y, Ishii T, Iida M and Sueishi K. Angiogenesis and lymphangiogenesis and expression of lymphangiogenic factors in the atherosclerotic intima of human coronary arteries. Hum Pathol. 2005;36:330-340.

[16] Takacs EJellinek H. Lymphatics in the aorta of rats treated with a soy-bean oil extract (lipofundin). Lymphology. 1986; 19:161-167.

[17] Davis JWinlove CP. Uptake of low molecular weight materials in an in situ preparation of the rabbit thoracic aorta. Artery. 1981;9:437-455.

[18] Eliska O, Eliskova M and Miller AJ. The morphology of the lymphatics of the coronary arteries in the dog. Lymphology. 1999;32:45-57.

[19] Eliska O, Eliskova M and Miller AJ. The absence of lymphatics in normal and atherosclerotic coronary arteries in man: a morphologic study. Lymphology. 2006;39:76-83.

[20] Galili O, Herrmann J, Woodrum J, Sattler KJ, Lerman LO and Lerman A. Adventitial vasa vasorum heterogeneity among different vascular beds. J Vasc Surg. 2004;40:529-535.

[21] Halin C, Tobler NE, Vigl B, Brown LF and Detmar M. VEGF-A produced by chronically inflamed tissue induces lymphangiogenesis in draining lymph nodes. Blood. 2007; 110:3158-3167.

[22] Greco KV, Lara PF, Oliveira-Filho RM, Greco RV and SudoHayashi LS. Lymphatic regeneration across an incisional wound: inhibition by dexamethasone and aspirin, and acceleration by a micronized purified flavonoid fraction. Eur $J$ Pharmacol. 2006;551:131-142.

[23] Bobryshev YV, Lord RS. Mapping of vascular dendritic cells in atherosclerotic arteries suggests their involvement in local immune-inflammatory reactions. Cardiovasc Res. 1998;37: 799-810. 